\title{
Pattern orientation in finite domains without boundaries
}

\author{
Lisa Rapp, Fabian Bergmann and Walter Zimmermann \\ Theoretische Physik I, Universität Bayreuth, 95440 Bayreuth, Germany
}

PACS 89.75.Kd - Pattern Formation

PACS 87.18. - $\mathrm{h}$ - Biological complexity

PACS 47.20.-k- Fluid instabilities

\begin{abstract}
We investigate the orientation of nonlinear stripe patterns in finite domains. Motivated by recent experiments, we introduce a control parameter drop from supercritical inside a domain to subcritical outside without boundary conditions at the domain border. As a result, stripes align perpendicular to shallow control parameter drops. For steeper drops, non-adiabatic effects lead to a surprising orientational transition to parallel stripes with respect to the borders. We demonstrate this effect in terms of the Brusselator model and generic amplitude equations.
\end{abstract}

Introduction. - Pattern formation is central to the wealth of fascinating phenomena in nature. It occurs in a great variety of physical, chemical and living systems [1]2]. Examples include patterns in isotropic and anisotropic ' convection systems [3,7], chemical reactions [8, 9] and biological systems [10 12, or environmental patterns [13.

In real systems, patterns emerge in finite areas or volumes. Consequently, spatially periodic patterns only contain a finite number of wavelengths. Along the system , borders, the relevant fields have to obey boundary condi' tions that influence the pattern in different ways 3 , 14 23 . ' In isotropic systems, they render the orientation of stationary patterns 3] 14. In thermal convection, convection rolls align perpendicular to side walls due to boundary conditions for the flow fields 15 17. Boundary conditions at ' the side walls may also restrict the range of possible stable wave numbers of periodic patterns [18. Traveling waves of finite wave number may be reflected at the boundaries leading to a number of interesting and complex phenomena [19] 23].

However, finite systems can also be achieved when the fluxes and forces driving a pattern, the so-called control parameters, are sufficiently strong (supercritical) only in a subdomain of the system. In this case, no specific boundary conditions act on the fields at control parameter drops to subcritical values. The effects of restricting patterns to a finite domain in this way have not been systematically investigated before. Examples of pattern orientations resulting from different widths of the control parameter drops are shown in fig. 1 and explained in this work. a)
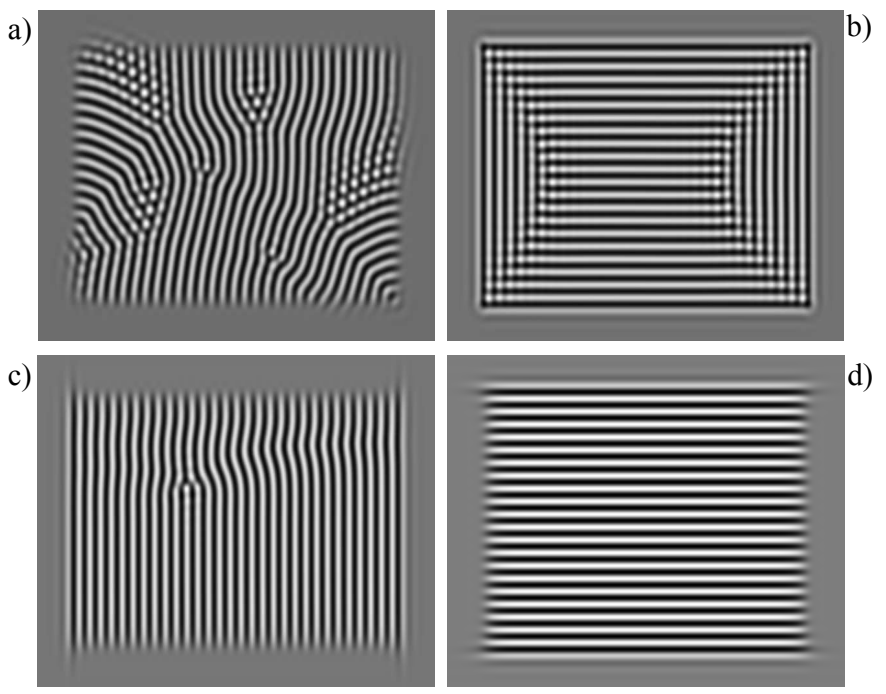

Fig. 1: Stripe patterns inside supercritical subdomains in the Brusselator model. The control parameter drops on different length scales $\delta_{x, y}$ along $x$ and $y$ from $\beta_{m}=0.05$ to subcritical values in a wide vicinity: a) $\delta_{x}=\delta_{y}=\lambda_{c}$, b) $\delta_{x}=\delta_{y}=0.32 \lambda_{c}$, c) $\delta_{x}=0.32 \lambda_{c}, \delta_{y}=1.5 \lambda_{c}$, d) $\delta_{x}=1.5 \lambda_{c}, \delta_{y}=0.32 \lambda_{c}$.

Recent experiments where pattern forming protein reactions take place in finite subdomains of substrates [24] belong to this class. Control parameter drops can also be designed in light sensitive chemical reactions where illumination of the reaction cell suppresses pattern formation 25, 26]. If the illumination is only applied to a subdomain of the system, again no boundary conditions for the concentration fields are defined along the edge of the illu- 
mination mask.

We investigate how control parameter drops along the borders of a supercritical subdomain affect the orientation of stationary spatially periodic patterns when no boundary conditions for the fields are specified. We choose the Brusselator as a representative model system to study the influence of the control parameter drop width. This is complemented by studies of the so-called amplitude equations for supercritical bifurcations to spatially periodic patterns 3. As a general description for this class of patterns, the conclusions drawn from the amplitude equations emphasize the universality of our results.

For large drop widths, we find that stripes align perpendicular to the borders of the supercritical control parameter domain. By decreasing the length scale for the control parameter drop, we find a surprising orientational transition to stripes in parallel alignment. The analysis of the amplitude equations reveals additional non-adiabatic, resonance-like effects favouring parallel stripes.

\section{Model systems and control parameter drop. -}

Brusselator. The Brusselator is a common model for reaction-diffusion systems [27]30]. We use it as a prototype system for supercritical bifurcations to spatially periodic patterns (Turing patterns). It describes the nonlinear behaviour of the concentration fields $u(x, y, t)$ and $v(x, y, t)$ :

$$
\begin{aligned}
\partial_{t} u & =\nabla^{2} u+a-(b+1) u+u^{2} v, \\
\partial_{t} v & =D \nabla^{2} v+b u-u^{2} v,
\end{aligned}
$$

with the control parameter $b$ and constant parameters $a$, $D$. These equations have the homogeneous fixed point solution

$$
u_{h}=a, \quad v_{h}=b / a .
$$

Turing patterns with the critical wave number $q_{c}$ bifurcate from this basic state for control parameter values beyond its critical one $b_{c}$ 29, where

$$
b_{c}=(1+a \eta)^{2}, \quad q_{c}=\sqrt{a \eta},
$$

and $\eta:=\sqrt{1 / D}$. The relative distance $\beta$ of the control parameter from its critical value $b_{c}$ is given by

$$
b=b_{c}(1+\beta),
$$

i.e. $\beta_{c}=0$. Hexagons are typical for the Brusselator near the onset of Turing patterns. But in this work, we consider the special case $D=a^{2}$ where stripes are preferred at the onset [30. In this case, the critical wavelength of the stripes according to eq. (3) is $\lambda_{c}:=2 \pi / q_{c}=2 \pi$. We choose $a=4$ throughout this work.

Amplitude equations. The two concentration fields $u$ and $v$ may be combined to the vector field $\mathbf{w}(\mathbf{r}, t)=$ $(u(\mathbf{r}, t), v(\mathbf{r}, t))$. We write spatially periodic stripes with the wave vector $\mathbf{q}_{c}$ in the form 3,29

$$
\mathbf{w}(\mathbf{r}, t)=\mathbf{w}_{h}+A \tilde{\mathbf{w}} \mathrm{e}^{\mathrm{i}\left(\mathbf{q}_{c} \cdot \mathbf{r}\right)}+A^{*} \tilde{\mathbf{w}}^{*} \mathrm{e}^{-\mathrm{i}\left(\mathbf{q}_{c} \cdot \mathbf{r}\right)},
$$

where $\mathbf{w}_{h}=\left(u_{h}, v_{h}\right)$. Slow variations (compared to the wavelength $\lambda_{c}$ ) of the envelope $A(\mathbf{r}, t)$ can be described by a dynamical amplitude equation [3, 31.

The Brusselator model is isotropic. Hence, in extended systems only the magnitude $q_{c}$ of the critical wave vector $\mathbf{q}_{c}$ for Turing stripes is fixed, but not its direction. Thus, all stripe orientations are equally likely at pattern onset. We consider the amplitude equations in two limits of stripe orientations: $\mathbf{q}_{c}=\left(q_{c}, 0\right)$ and $\mathbf{q}_{c}=\left(0, q_{c}\right)$, called parallel and perpendicular hereafter. The reduction method to amplitude equations, the so-called multiple scale analysis, is well established for supercritical bifurcations 3,31. The generic amplitude equations for the two stripe orientations in the case of a small and constant control parameter $\beta$ are

$$
\partial_{t} A=\beta A+\mathcal{L} A-g|A|^{2} A,
$$

with

$$
\begin{aligned}
& \mathcal{L}=\mathcal{L}_{\|}^{2}:=\xi_{0}^{2}\left(\partial_{x}-\frac{\mathrm{i}}{2 q_{c}} \partial_{y}^{2}\right)^{2} \quad \text { for } \quad \mathbf{q}_{c}=\left(q_{c}, 0\right), \\
& \mathcal{L}=\mathcal{L}_{\perp}^{2}:=\xi_{0}^{2}\left(\partial_{y}-\frac{\mathrm{i}}{2 q_{c}} \partial_{x}^{2}\right)^{2} \quad \text { for } \quad \mathbf{q}_{c}=\left(0, q_{c}\right) .
\end{aligned}
$$

The coherence length $\xi_{0}$ and the nonlinear coefficient $g$ for the Brusselator in the special case of $D=a^{2}$ are $\xi_{0}^{2}=1$ and $g=3 /\left(2 a^{2}\right)$ 30.

Control parameter drop. We introduce the control parameter drop by assuming the spatially dependent control parameter $\beta\left(x, \delta_{x}\right)$ :

$$
\beta=\beta_{0}+\frac{M}{2}\left[\tanh \left(\frac{x-x_{l}}{\delta_{x}}\right)-\tanh \left(\frac{x-x_{r}}{\delta_{x}}\right)\right] .
$$

We assume $L:=x_{r}-x_{l} \gg \lambda_{c}$ and $\beta_{0}<0 . \quad M$ and $\beta_{0}$ are chosen such that the maximum value $\beta_{m}=\beta_{0}+M$ is small and positive. Then $\beta\left(x, \delta_{x}\right)$ is supercritical in the subdomain $\bar{x}_{l}<x<\bar{x}_{r}$, where

$$
\bar{x}_{l, r}=x_{l, r} \pm \frac{\delta_{x}}{2} \ln \left(\frac{-\beta_{0}}{M+\beta_{0}}\right),
$$

and drops down to the subcritical value $\beta_{0}$ outside this domain. The steepness of the control parameter drop around $\bar{x}_{l, r}$ increases with decreasing values of the drop width $\delta_{x}$.

For small values of $\delta_{x}$, the control parameter $\beta\left(x, \delta_{x}\right)$ varies rapidly in a narrow range around $\bar{x}_{l, r}$. However, only the slowly (adiabatically) varying contributions to $\beta\left(x, \delta_{x}\right)$ affect the solutions of amplitude equations. The rapidly (non-adiabatically) varying part is smoothed out and must be treated separately. We therefore decompose $\beta\left(x, \delta_{x}\right)$ into an adiabatic and non-adiabatic part. For this purpose, we introduce the slow length scale $\delta_{A}:=$ $2 \xi_{0} / \sqrt{\beta_{m}}>\delta_{x}$ and choose $\beta_{0}=-\varepsilon, M=2 \varepsilon$ (where $\varepsilon$ is positive and small). We then express the slowly varying contribution $B_{0}(x)$ via eq. (8) by choosing $\delta_{x}=\delta_{A}$ :

$$
B_{0}(x)=\beta\left(x, \delta_{A}\right) .
$$


The difference between $\beta\left(x, \delta_{x}\right)$ and $B_{0}(x)$ becomes small in the centre of $\left[x_{l}, x_{r}\right]$ and takes its largest values around $x_{l, r}$. We expand the rapidly varying difference $\beta\left(x, \delta_{x}\right)-B_{0}(x)$ into a series to obtain

$$
\begin{aligned}
\beta\left(x, \delta_{x}\right)=B_{0}(x) & +\frac{M}{2} \sum_{m}\left\{B_{m}^{l}(x) \sin \left[m q_{c}\left(x-x_{l}\right)\right]\right. \\
& \left.+B_{m}^{r}(x) \sin \left[m q_{c}\left(x-x_{r}\right)\right]\right\}
\end{aligned}
$$

where $m=n / N_{L}, N_{L}=L / \lambda_{c}$. The integer $n$ is chosen such that $m$ belongs to the range $1 / 8,1 / 7 \ldots 4$. The functions $B_{m}^{l, r}(x)$ are localised around $x_{l, r}$ and represented by Gaussians of the form,

$$
B_{m}^{l, r}(x)=\hat{B}_{m}^{l, r} \exp \left[-\frac{\left(x-x_{l, r}\right)^{2}}{\delta_{G, m}^{2}}\right] .
$$

The Gaussian amplitudes $\hat{B}_{m}^{l, r}$ and their widths $\delta_{G, m}$ are determined via a correlation analysis. We calculate the correlation function between the rapidly varying part

$$
\Delta \tilde{\beta}(x, \delta)=\tanh (x / \delta)-\tanh \left(x / \delta_{A}\right)
$$

and the test function

$$
f_{m}\left(x, \delta_{\text {test }}\right)=\frac{1}{\sqrt{\pi} \delta_{\text {test }}} \mathrm{e}^{-x^{2} / \delta_{\text {test }}^{2}} \sin \left(m q_{c} x\right) .
$$

We then choose the Gaussian width $\delta_{G, m}$ to be the value of $\delta_{\text {test }}$ that maximises the correlation function. The amplitudes $\hat{B}_{m}^{l, r}$ are calculated via the overlap integral between $f_{m}\left(x, \delta_{G, m}\right)$ and $\Delta \tilde{\beta}$. Figure $\left.2 \mathrm{a}\right)$ shows the contributions $\bar{B}_{m}^{l}:=\varepsilon B_{m}^{l}(x) \sin \left(m q_{c} x\right)$ for $m=1,2$ in comparison to the full shape of $\beta\left(x, \delta_{x}\right)$. Both functions are localised around $x_{l}=0$ and approach zero within a short range $\left(\ll \delta_{A}\right)$ around the control parameter drop. The Gaussian amplitudes $\hat{B}_{1}^{l, r}$ and $\hat{B}_{2}^{l, r}$ decrease as a function of the drop width $\delta_{x}$ [fig. 2b)]. These non-adiabatic contributions vanish for $\delta_{x}>\delta_{A}$. The amplitude $\hat{B}_{1}^{l, r}$ is usually larger than $\hat{B}_{2}^{l, r}$, except in the limit of very small drop widths.

The patterns in fig. 1 are obtained for a rectangular supercritical subdomain of the control parameter in the form

$$
\begin{aligned}
\beta=\beta_{0}+\frac{M}{4} & {\left[\tanh \left(\frac{x-x_{l}}{\delta_{x}}\right)-\tanh \left(\frac{x-x_{r}}{\delta_{x}}\right)\right] } \\
\times & {\left[\tanh \left(\frac{y-y_{b}}{\delta_{y}}\right)-\tanh \left(\frac{y-y_{t}}{\delta_{y}}\right)\right] . }
\end{aligned}
$$

Here, we introduced a second drop width $\delta_{y}$ to describe the additional spatial dependence of $\beta$ in the $y$-direction. $\beta\left(x, y, \delta_{x}, \delta_{y}\right)$ is supercritical in the two-dimensional area $\left[\bar{x}_{l}, \bar{x}_{r}\right] \times\left[\bar{y}_{b}, \bar{y}_{t}\right]$.

Non-adiabatic effects cause an orientational transition. - We now include the control parameter drop into the amplitude equation using the decomposition

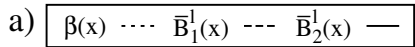

b) $\hat{\mathrm{B}}_{1}^{1, \mathrm{I}-\mathrm{N}} \hat{\mathrm{B}}_{2}^{\mathrm{l,r}}-$
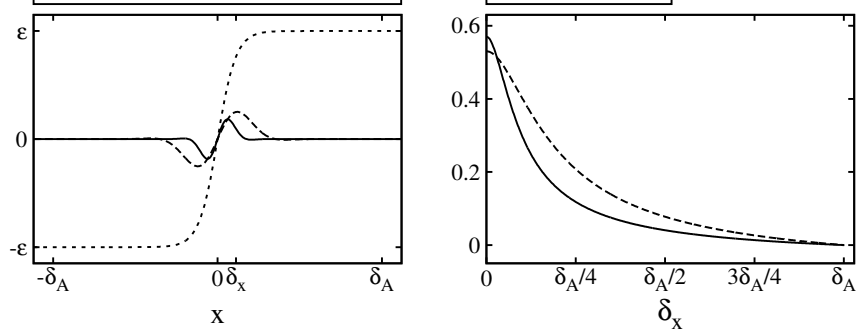

Fig. 2: a) Contributions $\bar{B}_{1}^{l}(x)$ and $\bar{B}_{2}^{l}(x)$ to the control parameter drop $\beta\left(x, \delta_{x}\right)$ for $\delta_{x}=0.11 \delta_{A}$. b) Gaussian amplitudes $\hat{B}_{1}^{l, r}$ and $\hat{B}_{2}^{l, r}$ of the localised amplitudes as a function of the drop width $\delta_{x}$ for $\varepsilon=0.05$.

given in eq. (11). The control parameter $\beta$ in eq. (6) is replaced by the slowly (adiabatically) varying part $B_{0}(x)$ as given by eq. (10). The short wavelength contributions $\propto B_{m}^{l, r}(x) \exp \left(i m q_{c} x\right)(m=1,2,3,4)$ in eq. (11) cause additional (non-adiabatic) terms in the amplitude equation for parallel stripes 32. It then takes the form

$$
\begin{aligned}
\partial_{t} A= & B_{0}(x) A+\mathcal{L}_{\|}^{2} A-g|A|^{2} A \\
& +\sum_{m=1}^{4} \alpha_{m} B_{m}(x)\left(A^{*}\right)^{m-1} .
\end{aligned}
$$

Here, $\alpha_{m}$ are constant parameters depending on the respective system. The complex localised contributions $B_{m}(x)$ due to the control parameter drop are given by

$$
B_{m}(x)=\mathrm{i} \frac{M}{4}\left[B_{m}^{l}(x) \mathrm{e}^{-\mathrm{i} m q_{c} x_{l}}-B_{m}^{r}(x) \mathrm{e}^{-\mathrm{i} m q_{c} x_{r}}\right] .
$$

The effects caused by $B_{3,4}(x)$ are much smaller than $B_{1,2}(x)$ and therefore neglected in the following. Equation (16) can be derived from the functional

$$
\begin{aligned}
F_{\|}= & \int d x d y\left[-B_{0}(x)|A|^{2}+\frac{g}{2}|A|^{4}+\left|\mathcal{L}_{\|} A\right|^{2}\right. \\
& \left.-\sum_{m=1}^{2} \frac{\alpha_{m}}{m}\left(B_{m}(x) A^{*^{m}}+B_{m}^{*}(x) A^{m}\right)\right]
\end{aligned}
$$

via $\partial_{t} A=-\delta F_{\|} / \delta A^{*}$. For the Brusselator in the case $D=a^{2}$, we find $\alpha_{1}=2 a$ and $\alpha_{2}=5 / 3$.

The amplitude equation for perpendicular stripes with $\mathbf{q}_{c}=\left(0, q_{c}\right)$ is not affected by resonance contributions $\propto$ $B_{m}$. It is described by eq. (6) with $\mathcal{L}=\mathcal{L}_{\perp}^{2}$ as given in eq. (7b) and the slowly varying control parameter $\beta=$ $B_{0}(x)$, cf. eq. (10). The related functional is

$$
F_{\perp}=\int d x d y\left[-B_{0}(x)|A|^{2}+\frac{g}{2}|A|^{4}+\left|\mathcal{L}_{\perp} A\right|^{2}\right] .
$$

Considering small values of $\delta_{x}$, non-adiabatic effects described by $B_{1,2}$ play an important role. However, these only affect the amplitude equation for parallel stripes, cf. eq. (16). A finite value of $B_{1}$ changes the supercritical 


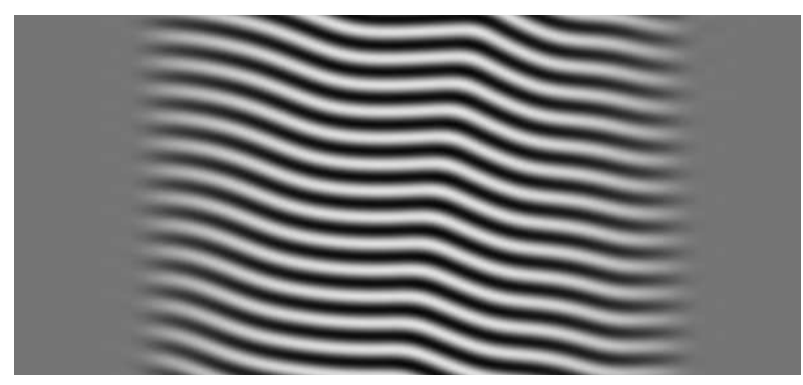

Fig. 3: Stripes favour a perpendicular orientation with respect to slow control parameter drops $\left(\delta_{x}=5 \lambda_{c}\right)$. Simulation of Brusselator started at $\beta_{m}=0.001$ and was slowly increased to $\beta_{m}=0.05$. Parameters: $l_{x}=l_{y}=50 \lambda_{c}, N_{x}=N_{y}=1024$. Note: Only a cutout of the simulation is shown.

pitchfork bifurcation (in the case $B_{1}=0$ ) to an imperfect bifurcation [32,33. Consequently, parallel stripes already have a finite amplitude below the bulk threshold $\beta_{m}=0$, especially around $x_{l, r}$, where $B_{1,2}$ take the largest values. This finite amplitude $A$ decreases the functional $F_{\|}$for parallel stripes with respect to $F_{\perp}$. Thus, for small values of $\delta_{x}$, parallel stripes are preferred compared to perpendicular stripes.

For large values of $\delta_{x}$, the non-adiabatic contributions $B_{1,2}$ become small and can be neglected (see fig. 2). In this case, the amplitude equations and the functionals for the two different stripe orientations only differ in the linear operator. These include different orders of derivatives in $x$-direction: $\left|\partial_{x} A\right|^{2}$ in the functional for parallel stripes, eq. (18), and $\left|\partial_{x}^{2} A\right|^{2}$ for perpendicular stripes, eq. (19). Thus, spatial variations of the amplitude $A(\mathbf{r}, t)$ affect the two functionals differently. The slow spatial variation of the control parameter $B_{0}(x)$ in $x$-direction is reflected in a spatial variation of the amplitude $A(\mathbf{r}, t)$. This increases both functionals. However, due to the different orders of $x$-derivatives, the functional for perpendicular stripes has a lower value 3,34 . Therefore, perpendicular stripes will be preferred for large $\delta_{x}$.

According to this reasoning, we predict stripes aligned perpendicular to the supercritical border for a large drop width $\delta_{x}$ and parallel for small $\delta_{x}$. Therefore, we expect an orientational transition for medium values of $\delta_{x}$. Note, for these considerations only the contributions $B_{0}, B_{1}$ and $B_{2}$ to the decomposition in eq. (11) are taken into account. However, the predicted orientational transition of stripes is rather insensitive to these approximations as confirmed by simulations of the Brusselator in the next section.

Numerical results for the Brusselator. - In the previous part we found an orientational transition of stripe patterns by changing the width of control parameter drops. This prediction is based on a reasoning including approximations. Therefore, the effect is verified by simulations of the Brusselator model, cf. eqs. (1), with supercritical subdomains of width $L=20 \lambda_{c}$, embedded in larger subcritical domains with overall system sizes $l_{x, y}$. The
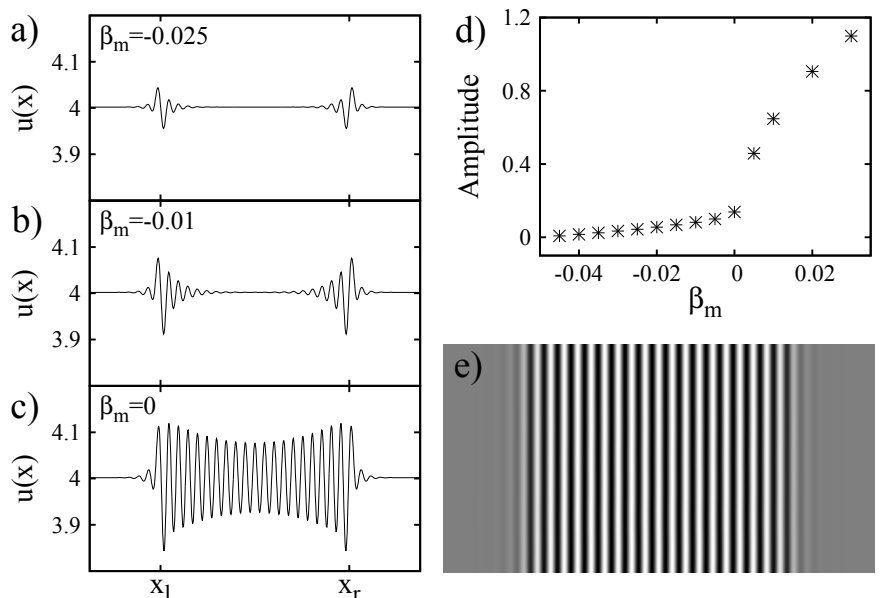

Fig. 4: Simulations of the Brusselator model with a narrow control parameter drop $\left(\delta_{x}=0.5 \lambda_{c}\right)$. Cross sections of the twodimensional stripe pattern for a) $\beta_{m}=-0.025$, b) $\beta_{m}=-0.01$, c) $\beta_{m}=0$. d) The stripe amplitude as a function of $\beta_{m}$ implies an imperfect bifurcation. e) Snapshot of the parallel stripes for $\beta_{m}=0.02$. Simulation parameters: $l_{x}=50 \lambda_{c}, l_{y}=25 \lambda_{c}$, $N_{x}=1024, N_{y}=512$.

model is solved using a common pseudospectral method with periodic boundary conditions 35 and $N_{x, y}$ modes, respectively. We choose $\beta_{0}=-0.05$ and perturb the basic solution by small amplitude random noise.

For large widths $\delta_{x}$ of control parameter drops, i.e. slow variations of the control parameter, the preferred orientation of a stripe pattern is nearly perpendicular to the borders of the supercritical domain, i.e. $\mathbf{q} \sim\left(0, q_{c}\right)$, as shown in fig. 3 for $\delta_{x}=5 \lambda_{c}$. This confirms the prediction in terms of the amplitude equations in the previous section (for bulk effects see Ref. [36]). Similar orientations are obtained for drop widths down to about $\delta_{x} \simeq \lambda_{c}$.

For small $\delta_{x}$, e.g. $\delta_{x}=0.5 \lambda_{c}$, the stripes align parallel to the borders of the supercritical range, i.e. $\mathbf{q}_{c} \sim\left(q_{c}, 0\right)$,

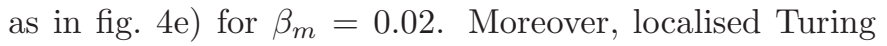
stripe patterns of finite amplitude occur around the borders at $x_{l, r}$ already at subcritical values of $\beta_{m}$ [see cross sections in fig. 将) and b)]. For increasing $\beta_{m}$, they expand into the whole supercritical domain. At the bulk

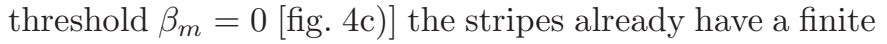
amplitude throughout the range $\left[x_{l}, x_{r}\right]$. The maximum stripe amplitude of the stationary solution as a function of $\beta_{m}$ is shown in the bifurcation diagram in fig. $4 \mathrm{~d}$ ). The form of the bifurcation is imperfect, as expected from the analysis on the basis of the amplitude equations in the previous section.

The two different preferred stripe orientations for large $\delta_{x}=5 \lambda_{c}$ in fig. 3 and small $\delta_{x}=0.5 \lambda_{c}$ in fig. 4 clearly confirm an orientational transition of stripes in the supercritical domain depending on the width of the control parameter drop along its border.

We can further restrict the domain size by varying the control parameter simultaneously along the $x$ - and $y$ - 


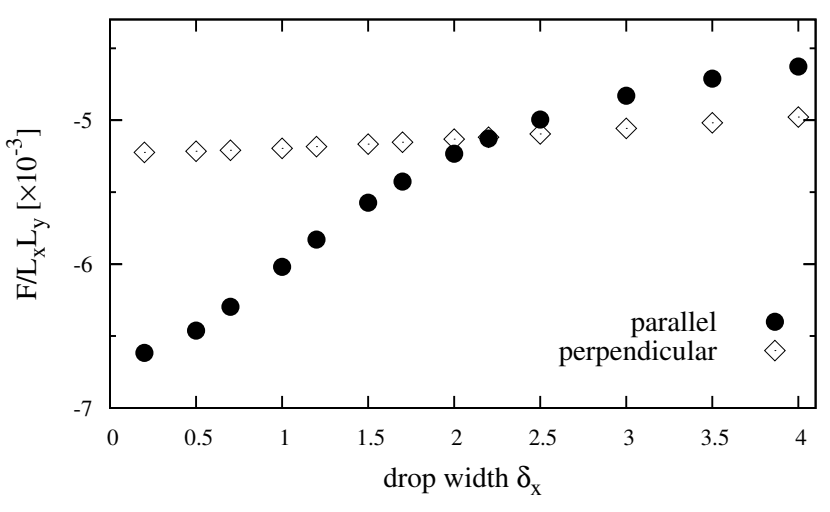

Fig. 5: Comparison of the functional for stripes as function of the drop width $\delta_{x}$ with the stripe wave vector $\mathbf{q}_{c}=\left(0, q_{c}\right)$ (filled circles) and $\mathbf{q}_{c}=\left(q_{c}, 0\right)$ (open diamonds). Parameters: $\beta_{0}=-0.05, M=0.1$.

direction, cf. eq. (15). In these rectangular domains [37, one can combine different drop widths $\delta_{x}$ and $\delta_{y}$ to trigger different stripe orientations as shown by four examples in fig. 1. Combining, e.g., large drop widths at the long side of the rectangle with small drop widths at the short side creates a remarkably uniform stripe pattern, cf. fig. [1). Using different combinations of $\delta_{x, y}$ may be a promising tool for designing Turing patterns in localised light sensitive chemical reactions 38 .

Orientational transition regime. - The orientational transition of stripes is deduced in terms of amplitude equations and confirmed by numerical simulations of the Brusselator model. The amplitude equations can be derived from the functionals, eqs. (18) and (19). Calculating these functionals as a function of the drop width allows to determine the preferred orientation for this $\delta_{x}$. In the range where $F_{\perp}<F_{\|}$, a perpendicular stripe orientation is expected and vice versa. For this purpose, we perform simulations of the amplitude equations for the two stripe orientations using the aforementioned pseudospectral algorithm (simulation parameters: $l_{x}=l_{y}=50 \lambda_{c}$, $\left.N_{x}=N_{y}=1024, L=20 \lambda_{c}, \beta_{0}=-0.05, \beta_{m}=0.05\right)$. When the solutions reach the stationary state, the functionals displayed in fig. 5 are calculated.

The functional corresponding to perpendicular stripes in eq. (19) does not contain the non-adiabatic contributions $B_{1}$ and $B_{2}$ to the control parameter drop. Regardless of the assumptions made for the justification of eq. (19) and the related amplitude equation, one may use $\beta\left(x, \delta_{x}\right)$ instead of $B_{0}(x)$. The functional then deviates only slightly from its constant value in the case of $B_{0}(x)$. In addition, fig. 5 shows that the functional with $\beta\left(x, \delta_{x}\right)$ is nearly independent of $\delta_{x}$, i.e. stripes perpendicular to the border of the supercritical range are rather insensitive to the width $\delta_{x}$.

For parallel stripes, $\mathbf{q}=\left(q_{c}, 0\right)$, the resonance effects covered by $B_{1}$ (and $B_{2}$ ) are relevant and the associated functional is given in eq. (18). The two functionals for the two different stripe orientations are shown as a function of the drop width $\delta_{x}$ in fig. 5. For narrow control parameter drops, i.e. $\delta_{x}$ small, the functional for parallel stripes is significantly lower. Thus, the parallel orientation is preferred. However, the functional for parallel stripes strongly increases as a function of the drop width. The orientational transition takes place at the intersection of the two functionals. For larger $\delta_{x}$, the perpendicular orientation of the stripes is preferred.

Summary and conclusions. - In this work, we identified and investigated a new class of finite pattern forming systems confined by control parameter drops from super- to subcritical values. These orient stripe patterns even without boundary conditions for the relevant fields. The stripe orientation depends on the width of the control parameter drops. We found a novel orientational transition of stripe patterns with respect to the borders as a function of the width of control parameter drops.

In light sensitive chemical reaction-diffusion systems showing Turing patterns [25,26] the transition length between the patterns (supercritical) and the homogeneous state (subcritical) may be varied by the length of a smooth transition between illuminated and dark areas.

The Swift-Hohenberg ( $\mathrm{SH}$ ) model 39] is besides the Brusselator a further paradigmatic model for studying the formation of spatially periodic patterns 2,3 . It behaves differently with respect to control parameter drops along the border of a supercritical domain. The basic state of the Brusselator is a function of the control parameter $b$, cf. eq. (2). Control parameter drops thus also change the basic state of the bifurcation to Turing patterns. In contrast, the basic state $u_{h}=0$ of the $\mathrm{SH}$ model remains unchanged for spatially varying control parameters. The same applies to the mean-field model for block copolymers (see i.e. 40). Therefore, we do not find the aforementioned orientational transition of stripe patterns in the $\mathrm{SH}$ or the block copolymer model. However, in common systems where the basic state is also changed by control parameter variations, orientational transitions of stripe pattern are very likely.

Our results for stationary patterns may also be important for traveling waves that occur for instance in the cell biological MinE/MinD protein reaction on flat substrates 12 24. To mimic the effects of cell confinement in such extended experiments, reactive membranes were created in subdomains of the substrate 24,41. In this way, the traveling waves are restricted to the range above the functionalised parts of the membrane. These may be interpreted as subdomains with a supercritical control parameter. In this experiment the traveling waves align perpendicular to the borders of the functionalised area 24]. It is very likely that this orientational behaviour is again governed by generic principles as discussed in this work and specific molecular reaction schemes or three-dimensional effects provide quantitative modifications [24,42,43]. Is the complex behavior of MinE/MinD oscillations in further 
restricted domains, as investigated recently in Ref. [44, determined by the specific properties of the kinetic reaction models? Or do again generic principles of pattern formation play a leading role as described in this work?

$$
* * *
$$

Enlightening discussions with M. Hilt and M. Weiß are gratefully acknowledged.

\section{REFERENCES}

[1] Ball P., The Self-Made Tapestry: Pattern Formation in Nature (Oxford Univ. Press, Oxford) 1998.

[2] Cross M. C. and Greenside H., Pattern Formation and Dynamics in Nonequilibrium Systems (Cambridge Univ. Press, Cambridge) 2009.

[3] Cross M. C. and Hohenberg P. C., Rev. Mod. Phys., 65 (1993) 851.

[4] LAPPA M., Thermal Convection: Patterns, Evolution and Stability (Wiley, New York) 2010.

[5] Weiss S., Seiden G. and Bodenschatz E., New J. Phys, 14 (2012) 053010.

[6] Bodenschatz E., Zimmermann W. and Kramer L., J. Phys. (Paris), 49 (1988) 1875.

[7] Kramer L. and Pesch W., Annu. Rev. Fluid Mech., 27 (1995) 515

[8] Kapral R. and Showalter K., (Editors) Chemical Waves and Patterns (Springer, New York) 1995.

[9] Mikhailov A. S. and Showalter K., Phys. Rep., 425 (2006) 79 .

[10] Ben-Jacob E., Shochet O., Tenenbaum A., Cohen I., Czirok A. and Vicsek T., Nature, 368 (1994) 46.

[11] Kondo S. and Miura T., Science, 329 (2010) 1616.

[12] Loose M., Fischer-Friedrich E., Ries J., Kruse K. and Schwille P., Science, 320 (2008) 789.

[13] Meron E., Nonlinear Physics of Ecosystems (Taylor \& Francis, Oxon, UK) 2015.

[14] Greenside H. and Coughran W. M., Phys. Rev. A, 30 (1984) 398.

[15] Cross M. C., Phys. Fluids, 25 (1982) 936.

[16] Bajaj K. M., Mukolobwiez N., Currier N. and Ahlers G., Phys. Rev. Lett., 83 (1999) 5282.

[17] Chiam K.-H., Paul M. R., Cross M. C. and GreenSIDE H., Phys. Rev. E, 67 (2003) 056206.

[18] Cross M. C., Daniels P. G., Hohenberg P. C. and SigGia E. D., J. Fluid Mech., 55 (1983) 155.

[19] Cross M. C., Phys. Rev. Lett., 57 (1986) 2935.

[20] Cross M. C., Phys. Rev. A, 38 (1988) 3593.

[21] Moses E., Fineberg J. and Steinberg V., Phys. Rev. A, 35 (1987) 2757.

[22] Kolodner P. and Surko C. M., Phys. Rev. Lett., 61 (1988) 842; Kolodner P., Phys. Rev. A, 44 (1991) 6448.

[23] Garnier N., Chiffaudel A. and Daviaud F., Physica D, 174 (2003) 30.

[24] Schweizer J., Loose M., Bonny M., Kruse K., MÖnch I. and Schwille P., Proc. Natl. Acad. Sci. (USA), 109 (2012) 15283.

[25] Munuzuri A. P., Dolnik M., Zhabotinsky A. M. and Epstein I. R., J. Am. Chem. Soc., 121 (1999) 8065.
[26] Dolnik M., Berenstein I., Zhabotinsky A. M. and Epstein I. R., Phys. Rev. Lett., 87 (2001) 238301.

[27] Prigogine I. and Lefever R., J. Chem. Phys., 48 (1969) 1695.

[28] Nicolis G. and Prigogine I., Self-Organization in Nonequilibrium Systems, from Dissipative and Order through Fluctuations (Wiley, New York) 1977.

[29] Walgraef D., Spatio-Temporal Pattern Formation (Springer, New York) 1997.

[30] Pena B. and Perez-Garcia C., Phys. Rev. E, 64 (2001) 056213.

[31] Newell A. C. and Whitehead J. A., J. Fluid Mech., 38 (1969) 279.

[32] Coullet P., Phys. Rev. Lett., 56 (1986) 724.

[33] Peter R. et AL. , Phys. Rev. E, 71 (2005) 046212.

[34] Malomed B. A. and Nepomnyashchy A. A., Europhys. Lett., 21 (1993) 195.

[35] Kopriva D. A., Implementing Spectral Methods for Partial Differentail Equations (Springer, Netherlands) 2009.

[36] Freund G., Pesch W. and Zimmermann W., J. Fluid Mech., 673 (2011) 318.

[37] Simulation parameters: $\beta_{0}=-0.1, \beta_{m}=0.05, L_{x}=$ $30 \lambda_{c}, L_{y}=20 \lambda_{c}, l_{x}=60 \lambda_{c}, l_{y}=50 \lambda_{c}, N_{x}=N_{y}=1024$,

[38] Steinbock O., Kettunen P. and Showalter K., Science, 269 (1995) 1857.

[39] Swift J. B. and Hohenberg P. C., Phys. Rev. A, 15 (1977) 319.

[40] Weith V., Krekhov A. and Zimmermann W., J. Chem. Phys., 129 (2013) 054908.

[41] Lagny T. J. and Bassereau P., Interface Focus, 5 (2015) 20150038.

[42] Halatek J. and Frey E., Cell Reports, 1 (2012) 741.

[43] Halatek J. and Frey E., Proc. Natl. Acad. Sci. USA, 111 (2014) E1817.

[44] Wu F., van Schie B. G. C., Keyner J. E. and Dekker C., Nature Nanotechnology, 10 (2015) 719. 